\title{
Adventures in Cooperative Learning: An Ongoing Experiment
}

\author{
Sandra A. Yost, P.E., Ph.D. \\ N. Mohankrishnan, Ph.D. \\ University of Detroit Mercy
}

\begin{abstract}
Global competition has and will continue to drive the ways in which U.S. companies do business. Increasingly, those who hire engineering graduates look for employees who are not only technically proficient, but who have also demonstrated leadership and initiative in team settings. Since much engineering work takes place in teams, cooperative learning is a useful paradigm for developing these skills in our students. Furthermore, participation in groups representing a diversity of backgrounds and cultures prepares the engineering graduate to function in the global arena.
\end{abstract}

Many studies argue that the use of formal cooperative learning groups in engineering courses has a positive effect on student outcomes. While the literature reports many successes with this teaching methodology, it is more difficult to find practical strategies for beginning to integrate cooperative learning groups in a significant way in courses that have traditionally been taught in a predominantly lecture mode.

This paper approaches the adoption of a formal cooperative learning component from a practical perspective. The first author has implemented a formal cooperative learning component in engineering courses taught in the last two years. The strategies discussed here result from these early attempts to make cooperative learning groups work, providing a useful guide for instructors who wish to incorporate this innovative teaching style into their own courses. The paper also discusses the results of a recent experiment in cooperative learning conducted by the co-authors.

\section{Introduction}

Cooperative learning can be defined as the "instructional use of small groups so that students work together to maximize their own and each others' learning." [1] Numerous studies, including [1-4] and many others, conclude that the proper use of cooperative learning techniques in the classroom has a positive effect on the students' mastery of important concepts. Students who participate in such groups also learn important lessons in teamwork, communication, and leadership. Such attention to the development of both technical and professional skills is of the same spirit articulated in the ASEE report titled "Engineering Education for a Changing World"[5].

In this paper, we describe an experiment designed to assess how well the incorporation of a formal cooperative learning component enhanced student learning in an engineering course. While the results are important and will be discussed, we feel that the practical lessons learned in developing this style of instruction will be of the most use to instructors who wish to use this 
strategy to enhance student enthusiasm for learning.

\section{Background}

The experiment described in this paper arose from the first author's initial experiences in incorporating a formal cooperative learning component in an introductory electronics course at the University of Detroit Mercy (UDM). Electronics I (EE 356) is a required course for sophomore Electrical Engineering students, and is offered in a single section. Since there was no control group, we could not make a quantitative determination of the effectiveness of cooperative learning in this course. A mid-semester survey was given to assess student attitudes towards this instructional paradigm, and adjustments were made. The results of this survey were discussed in [6]. At the end of the semester, students were required to write a brief reflection paper on how their cooperative learning group was or was not helpful to their learning in the course.

Although the Electronics I students reported a reasonable degree of satisfaction with the use of cooperative learning groups, the lack of comparative data on student performance in lecture vs. cooperative learning courses prevented us from declaring the initial experiment an unqualified success. An opportunity to explore this issue further came in the Summer of 1997, when two sections of Principles of Electrical Engineering (E 320) were offered to junior level non-EE students. Note that summer is the normal time for them to take this required course because of UDM's engineering co-op rotation schedule. The use of cooperative learning groups in nonmajor service courses presents a unique challenge, since many students in such courses are interested only in getting by with a minimum of effort.

Most recently (Fall 1997), Network Theory I (EE 350), the first EE course for EE majors, included a formal cooperative learning component. The lessons learned from the previous two experiences with this teaching strategy were incorporated into a style which while continuing to evolve, will provide a fundamental framework for future development of the techniques that comprise the style.

\section{The Experiment}

Two sections of E 320, Principles of Electrical Engineering, each accommodating roughly 30 students, met simultaneously during the summer term in 1997. Mohankrishnan's section was taught primarily in a traditional lecture format, while Yost used a formal cooperative learning component. The students did not self-select into lecture vs. cooperative learning, although one student switched out of the cooperative learning section after the first class meeting.

Because the course was taught using two different instructors, it was impossible to completely eliminate the effect of this variable, but we were able to minimize its effect significantly. First, because of scheduling, we were able to combine the two sections for the exams, giving the same exam to both groups. Each instructor wrote half of each exam, and graded that half for all students, so that grading would be consistent for the exams. We also met weekly to make sure that both sections received instruction for the same topics each week, and were assigned the same problem sets for homework. 
Since we believe that the composition of the groups is critical for the success of cooperative learning, an elaborate rotation process was used to allow all students to have the opportunity to work with every other student before being assigned. This allowed the students to learn if there was anyone in the class with whom they felt they could not work. (Most students did not identify anyone to avoid.) The groups of three or four were formed with an eye towards heterogeneity, although efforts were made to avoid isolating women and minority students as the only one in their groups. Course and work schedules were the primary consideration for forming the groups, since it was expected that the groups would work together outside of class on assignments, and it was hoped that they would help each other in preparing for exams. Each group had at least one relatively high-achieving student, based on incoming GPA.

The section taught using cooperative learning groups made liberal use of in-class group activities, which significantly reduced the time available for lecture. To keep on schedule, students were required to do pre-class reading assignments from the text, and "mini-lecture" time was used to summarize important points from the assignment and to work one or two examples before turning the groups loose on other sample problems. Group homework assignments did not completely replace individual assignments, but several of these gave the groups the opportunity to work together on some open-ended problems that required more time and thought than the 75minute class periods allowed.

Each group met with the instructor at least once during the semester to discuss their individual learning goals for the course and to discuss how the group was helping them to achieve these goals.

\section{Results}

The results of our experiment are summarized in Table 1 which makes a comparison between the performance of the cooperative learning (CL) group and the control group. The first column lists the mean GPA of the students in the two sections at the beginning of the course. Their average performance in the examination components of the course is presented in the remaining columns.

\begin{tabular}{|l|l|l|l|l|l|}
\hline & GPA(in) & Exam 1 & Exam 2 & Final Exam & Overall \\
\hline Mean (CL) & 3.08 & 68.9 & 55.9 & 55.7 & 66.8 \\
\hline Mean (Control) & 3.34 & 73.8 & 59.0 & 61.8 & 69.8 \\
\hline \% Difference & $7.8 \%$ & $6.6 \%$ & $5.3 \%$ & $9.9 \%$ & $4.0 \%$ \\
\hline
\end{tabular}

Note: \% Difference $=($ Control - CL)/Control x $100 \%$

Table 1: Results from E 320 Experiment

It should be noted that the average GPA of the students in the control group was higher than that of the students in the CL group by 7.8\%. But in Exam 1 and 2 they only did better by $6.6 \%$ and $5.3 \%$ respectively; the narrowing of the gap between the two groups could perhaps be attributed 
to the influence of the use of cooperative learning techniques. However, the Final Exam results buck this trend with a widening of the gap to $9.9 \%$. The overall score for the course (reflecting the composite of the homework and exam components) reflects a narrowing of the differential to about $4 \%$.

While the quantitative measures discussed above might be interpreted as mixed and inconclusive, we believe that it is important to withhold judgment on the use of cooperative learning techniques until some qualitative aspects are considered - one must remember that some of the claimed benefits of the use of such techniques have to do with the development of abstract skills such as teamwork, for instance, that cannot be adequately measured by the numbers discussed above. For this task, let us turn to the opinions expressed by the students in a reflection paper that they were required to write at the end of the Principles and Electronics courses. Selected student comments are presented below:

- The cooperative learning groups were helpful because they gave us an opportunity to attempt problems and/or projects that may prove to be discouraging as an individual effort.

- The one large benefit which I see in working with groups is that it more closely resembles a real work environment.

- I usually picked up the class material pretty well, but my knowledge was strengthened by having to explain myself to the rest of the group.

- It was not like the group did not have a basic knowledge on their own, but more like we all had different approaches to solve the same problem. Therefore, when we explained our individual ideas to the group, we got exposed to things we had not, and might not have thought of, thus expanding our knowledge.

- I think that the instructor should stick with these Cooperative Learning groups, because they are beneficial, whether or not students see it.

- $\quad$ The major benefits I found with group projects is that there is always someone to help motivate members to finish assignments and not to get discouraged.

- Ifelt the success of the group as my success too.

- Every member thinks differently and by putting everyone's ideas together, the task can be completed much quicker.

\section{Implications}

A number of lessons have been learned over the past two years of the authors' experience of using the cooperative learning paradigm in engineering courses. Here we share what we think are some of the most important practical observations for instructors who are interested in using cooperative learning groups in their courses. 
1. Composition of the groups is important. Do not give in to pressure from the students to choose their own groups. Without diversity, the benefits of learning to work with different people are not fully realized. Do take into account the times that students have available for group work outside of class. If necessary, let students choose their own groups for out-ofclass assignments, but use instructor-formed groups for in-class assignments.

2. Be prepared to help groups manage conflict. With diversity, conflict can arise within groups. Instructors need to have some basic skills in conflict resolution, and be able to teach this to groups.

3. Cooperative learning does not entirely replace lecture. Skillful instructors will address the entire class just enough to situate the day's topic in the proper context, and provide one or two carefully chosen examples to ensure that the groups will be able to function relatively autonomously in group activities. Do not try to "squeeze" the entirety of your former lectures into a shorter time frame!

4. Using an absolute grading scale is a necessity! Grading on a curve fosters competition, not cooperation.

5. Carefully planned group exercises achieve the best results. For out-of-class activities, it is important to structure the assignment so that the team members are motivated to rely on each other to successfully complete all of the tasks. It is often possible to modify a challenging text problem to include some computer simulation and analysis to make a non-trivial but doable group assignment. In-class group assignments pose more difficulties, because of time constraints and, in our experience, students' lack of preparation prior to class. If students can be motivated to prepare by reading the text material in advance (even pop-quizzes failed to provide sufficient motivation), they can be expected to solve less trivial problems in class. In any case, even simple drill exercises provide the students with some measure of confidence that they can apply the concepts before tackling homework assignments.

6. Exam bonus points can be useful in fostering cooperation. We find that it is rare for a group to become what the literature defines as a "base group". Most of our groups were able to work together to achieve a particular task. Almost none of them reported that they spent significant amounts of time studying together when no specific group assignment was due. In the Network Theory (EE 350) course offered in Fall, 1997, groups were offered bonus points if all of their members scored above a minimum percentage on individually-taken written exams. We found that this provided an incentive for the higher-achieving group members to assist those having some difficulty with important concepts. This was tried once near the end of the Principles of EE (E 320) course for non-EE's, but was received much more enthusiastically in the course for EE majors. In our judgment, several of the groups from the Network Theory course functioned as base groups as a result. The following anecdote illustrates the positive effect such a strategy can have:

After the first exam, one group found that one of the three group members did not make 
the minimum score for the group to earn bonus points. The other group members decided to make him the group leader for the next major group assignment so that he would be in a better position to understand all of the concepts required to complete the assignment, and thus qualify the group for a bonus on the next exam. For in-class assignments, this group also made a concerted effort to encourage their team member's active and informed participation. This resulted in some friction, due in some part to cultural differences in giving and interpreting encouragement, but the group worked through the difficulties without intervention by the instructor, and as a result, we believe they gained valuable experience in teamwork and communication.

7. An attendance policy is critical for courses taught in a cooperative learning format. After the first author's experience in Electronics I, it was clear that poor attendance by one or more group members was a major factor in some groups' dissatisfaction with cooperative learning. The attendance policy used currently allows students to miss three or four classes during the semester without penalty. After that, the student is removed from his or her group unless the group lobbies to keep the member. The student must still complete group exercises, but alone, and with a significant point penalty assessed on formal group assignments. This has relatively little direct impact on the student's grade for the course, but we find that it does, at least in the Network Theory course for EE majors, provide extra motivation to attend and participate in class. (In the Principles of EE course, 4 students out of 29 violated the attendance policy by the middle of the term, and were removed from their groups.)

\section{Conclusion}

Cooperative learning demands more of both the student and the instructor, but its incorporation in engineering courses can enhance student performance, especially among students who usually struggle in courses taught using the traditional lecture format. Whether or not higher achieving students benefit intellectually from this style depends largely on their ability and willingness to work cooperatively with team members who need help to grasp difficult concepts. For all students who make the effort to work productively in groups, important lessons in teamwork and communication skills are learned. The final reflection paper assigned at the end of the course asks students to assess how their experiences with cooperative learning can enhance their careers and make them more competitive in the job search. Because heterogeneity is a characteristic of the assigned groups, students learn important skills in working with people from different cultures, disciplines, and perspectives. Such skills in today's engineering workforce are critical for the global competitiveness of U.S. industry.

\section{References}

1. Smith, K. A., "Cooperative learning: effective teamwork for engineering classrooms," Proceedings, Frontiers in Education 25 $5^{\text {th }}$ Annual Conference, Vol. 1, pp. 2b5.13-18, Nov. 1995.

2. McDonald, D., "Integrating cooperative learning experiences in an instrumentation and control course," 1995 ASEE Annual Conference Proceedings, Vol. 1, pp. 807-813, June 1995.

3. Cavalier, J. C., Klein, J. D., Cavalier, F. J., "Effects of cooperative learning on performance, attitude, and group behaviors in a technical team environment," Educational Technology Research and Development, 
Vol. 43, No. 3, pp. 61-71, 1995.

4. Hilborn, R. B., "Team learning for engineering students," IEEE Transactions on Education, pp. 207-211, Aug. 1994.

5. ASEE Project Report, "Engineering education for a changing world," ASEE Prism, Vol. 4, Dec. 1994.

6. Yost, S. A., "Factors Affecting The Successful Integration of a Cooperative Learning Component in Classroom Instruction," Proceedings, ASEE 1997 North Central Section Conference, Vol. 1, pp. 154-159, April 1997.

\section{Biographical Information}

Sandra A. Yost is an Assistant Professor in the Department of Electrical and Computer Engineering at the University of Detroit Mercy. She is active in several professional societies, including IEEE, ASEE, and SWE. Her interests include discrete time system theory, control system applications, and engineering education.

N. Mohankrishnan is an Associate Professor in the Department of Electrical and Computer Engineering at the University of Detroit Mercy. He is a member of IEEE and ASEE. His research interests are in the areas of engineering education and Digital Signal Processing Applications. 